\title{
The Behaviour of the Eigenvalues for a Class of Operators Related to some Self-Affine Fractals in $\boldsymbol{R}^{2}$
}

\author{
W. Farkas
}

Abstract. The obtaining of sharp estimates for the asymptotic behaviour of the eigenvalues of the (semi-elliptic) operator acting in the anisotropic Sobolev space

$$
\dot{W}_{2}^{(1,2)}(\Omega)=\left\{u \in W_{2}^{(1,2)}(\Omega): u\left|\partial \Omega=\frac{\partial u}{\partial x_{2}}\right| \partial \Omega=0\right\}
$$

generated by the quadratic form $\int_{\Omega} f(\gamma) \overline{g(\gamma)} d \mu(\gamma)$ is investigated. Here $\mu$ is an appropriate self-affine fractal measure on the unit disc $\Omega \subset \mathbb{R}^{2}$.

Keywords: Regular anisotropic fractals, anisotropic function spaces, semi-elliptic differential operators

AMS subject classification: Primary 35 P 15 , secondary 46 E 35,28 A 90

\section{Introduction}

Fractal geometry is a very attractive and quickly developing field of modern mathematics. Thank to the work done by A. Jonsson and H. Wallin [18], K. Naimark and M. Solomyak [21, 22], H. Triebel [32], D. E. Edmunds and H. Triebel [9] it turns out that various aspects of this theory are closely connected to Fourier analysis, to the modern theory of function spaces and to spectral theory of partial differential equations. This paper has to be understood as a contribution in this direction. The purpose of our work is to highlight the connection between the asymptotic behaviour of the eigenvalues of some differential operators related to some fractals and the properties of the fractals.

Spectral properties and especially the distribution of the positive eigenvalues of fractal differential operators of the form

$$
U=(-\Delta)^{-1} \circ t r^{\Gamma}
$$

W. Farkas: Univ. Bundeswehr München, Inst. Theor. Inf. \& Math., Werner-Heisenberg-Weg 39, D-85577 Neubiberg. - This work was supported by the DFG Graduiertenkolleg "Analytical and Stochastical Structures and Systems" at the University of Jena and partly by the DFG project Ja522/7-1. It is a pleasure to give our warm thanks to Prof. H. Triebel for stimulating conversations.

ISSN 0232-2064 / \$2.50 C Heldermann Verlag Berlin 
were studied in $[9,21,22,32]$. Here $(-\Delta)^{-1}$ is the inverse of the Dirichlet Laplacian in the bounded $C^{\infty}$ domain $\Omega \subset \mathbb{R}^{n}, \Gamma$ is a compact fractal with

$$
\Gamma \subset \Omega, \quad|\Gamma|=0, \quad \operatorname{dim}_{H} \Gamma<n,
$$

and $t r^{\Gamma}$, closely related to the trace operator $t r_{\Gamma}$, is given by

$$
\left(\operatorname{tr}^{\Gamma} f\right)(\varphi)=\int_{\Gamma}\left(\operatorname{tr}_{\Gamma} f\right)(\gamma)(\varphi \mid \Gamma)(\gamma) d \mu(\gamma) \quad(\varphi \in D(\Omega))
$$

where $\mu$ is the Radon measure underlying the fractal $\Gamma$. The study of operators of type (1.1) is motivated in a natural way by the so-called fractal drums. If $\lambda_{k}(k \in \mathbb{N})$ are the positive eigenvalues of $(-\Delta)^{-1} \circ t r^{\Gamma}$, ordered by magnitude and counted with respect to their multiplicities, then $\eta_{k}=\lambda_{k}^{-\frac{1}{2}}$ are the eigenfrequencies of a vibrating membrane (interpreted as the bounded domain $\Omega$ ), fixed at its boundary, having the whole mass concentrated on the fractal $\Gamma \subset \Omega$. More details are given in [32: Subsections 26.2 and 30.1 - 30.5], where one can find a detailed discussion on different aspects concerning fractal drums.

Satisfactory results were obtained if $\Gamma$ is is a self-similar fractal (see $[21,22]$ ) and if $\Gamma$ is an (isotropic) $d$-set with $n-2<\operatorname{dim}_{H} \Gamma<n$ (see [32: Theorem 30.2]). Recall $\Gamma \subset \mathbb{R}^{2}$ is an isotropic $d$-set if there are a Radon measure $\mu$ in $\mathbb{R}^{2}$ and two positive numbers $c_{1}$ and $c_{2}$ such that supp $\mu=\Gamma$ and, for all balls $B(\gamma, r)$ centred at $\gamma \in \Gamma$ and of radius $0<r<1, c_{1} r^{d} \leq \mu(B(\gamma, r)) \leq c_{2} r^{d}$. Self-similar fractals are typical examples of (isotropic) $d$-sets. The notion of (isotropic) $d$-set occurs both in the theory of function spaces and in fractal geometry (see $[10,11,18,32])$.

For anisotropic fractals in $\mathbb{R}^{2}$ with (1.2) as defined in [32: Definition 5.2] one has for the eigenvalues of $U$ only two-side estimates of type

$$
c_{1} k^{-\omega_{1}} \leq \lambda_{k}(U) \leq c_{2} k^{-\omega_{2}} \quad(k \in \mathbb{N})
$$

for appropriate positive numbers $\omega_{1}$ and $\omega_{2}$ with $\omega_{1} \geq 1 \geq \omega_{2}$ (see [32: Theorem 30.7]). Improvements of (1.4) were discussed in [14]. However, the exponents in (1.4) are not equal in the general case (see the precise formulation in [32: Theorem 30.7] and cf. [14: Theorem 3.1]). This fact should be not a big surprise since one can imagine that the different nature of the (isotropic) operator $-\Delta$ and the (anisotropic) structure of $\Gamma$ would cause difficulties (see also the discussion in [32: Subsection 4.16]).

Let $0<d<2$ and let $a=\left(a_{1}, a_{2}\right)$ with $0<a_{2} \leq a_{1}<\infty, a_{1}+a_{2}=2$. A regular anisotropic $d$-set with respect to the anisotropy $a$ is, roughly speaking, a compact set $\Gamma \subset \mathbb{R}^{2}$ which can be covered for any $j \in \mathbb{N}_{0}$ with $N_{j} \sim 2^{j d}$ disjoint rectangles having sides parallel to the axes and with side lengths $r_{1}^{j, l}$ and $r_{2}^{j, l}\left(l=1, \ldots, N_{j}\right)$ satisfying $r_{i}^{j, l} \sim 2^{-j a_{i}}$ for $i=1,2$ (the equivalence constants being independent of $j, l$ and $i$; cf. [32: Subsection 4.18] and see the precise definition in Section 2). Those fractals are special cases of the anisotropic fractals introduced in [32: Definition 5.2].

Considering $\Gamma$ a regular anisotropic $d$-set with respect to the anisotropy $a=\left(a_{1}, a_{2}\right)$, our intention is here to replace the Dirichlet Laplacian $-\Delta$ in (1.1) with a model semielliptic differential operator $A$ whose "anisotropic structure" coincides with that of the 
fractal $\Gamma$. We will investigate spectral properties of the new operator $A^{-1}$ otr $r^{r}$ acting in an appropriate anisotropic Sobolev space $\dot{W}_{2}^{\left(s_{1}, s_{2}\right)}(\Omega)$ being interested mainly in the possibility of obtaining estimates of the type $\lambda_{k}\left(A^{-1} \circ t r^{\Gamma}\right) \sim k^{-\omega}(k \in \mathbb{N})$ (equivalence constants independent of $k \in \mathbb{N}$ ) for its eigenvalues, where $\omega$ is an appropriate positive number.

Let $\Omega=\left\{x=\left(x_{1}, x_{2}\right) \in \mathbb{R}^{2}: x_{1}^{2}+x_{2}^{2}<1\right\}$ be the unit disc in the plane and let $\partial \Omega$ be its boundary. In order to extend the theory of regular elliptic operators to more general classes of operators, H. Triebel considered in [28] the semi-elliptic differential operator

$$
\left(\mathcal{A}_{r} u\right)(x)=-\frac{\partial^{2} u(x)}{\partial x_{1}^{2}}+\frac{\partial^{4} u(x)}{\partial x_{2}^{4}}+\frac{r}{\eta^{2}\left(x_{1}\right)} u(x) \quad\left(x=\left(x_{1}, x_{2}\right) \in \Omega\right)
$$

where $r \in \mathbb{R}$ and $\eta$ is a $C^{\infty}$-function on the interval $[-1,1]$ with $\eta(t)>0$ if $|t|<1$ and

$$
\lim _{t \uparrow 1} \frac{\eta(t)}{1-t}=\lim _{t \downarrow-1} \frac{\eta(t)}{1+t}=1
$$

and a corresponding boundary value problem $\mathcal{A}_{r} u(x)=f(x)$ if $x \in \Omega, u(y)=g_{1}(y)$ (y $\epsilon$ $\partial \Omega)$ and $\frac{\partial u}{\partial x_{2}}(y)=g_{2}(y)(y \in \partial \Omega)$ where $f, g_{1}$ and $g_{2}$ are given functions. In [28] the author obtained a-priori estimates for the operator $\mathcal{A}_{r}$ in the framework of the anisotropic Besov spaces $B_{p p}^{(s, 2 s)}\left(\Omega, x^{0}, x^{1}\right)$ and the anisotropic Sobolev spaces $W_{p}^{(s, 2 s)}\left(\Omega, x^{0}, x^{1}\right)$ where $1<p<\infty$. These two scales of spaces (our notation here is slightly different from the original one in [28] where they were denoted $\dot{B}_{p p}^{(s, 2 s)}(\Omega)$ and $\dot{W}_{p}^{(s, 2 s)}(\Omega)$ ) were considered in [29] taking into account the singular points $x^{0}=(-1,0)$ and $x^{1}=(1,0)$. They are close to the anisotropic spaces on $\Omega$ but their elements show (roughly speaking) a peculiar behaviour near the singular points $x^{0}$ and $x^{1}$. A description of this theory may be found also in [24: Section 4.8].

Spectral properties of $\mathcal{A}_{r}$ acting as an unbounded operator defined in $L_{p}(\Omega) \quad(1<$ $p<\infty)$ having domain of definition $\mathcal{D}\left(\mathcal{A}_{r}\right)=\left\{u \in W_{p}^{(2,4)}\left(\Omega, x^{0}, x^{1}\right): u \mid \partial \Omega=\right.$ $\left.\frac{\partial u}{\partial x_{2}} \mid \partial \Omega=0\right\}$ (see the proof of Theorem 4.2 below for the precise definition of the space $W_{p}^{(2,4)}\left(\Omega, x^{0}, x^{1}\right)$ if $\left.p=2\right)$ were discussed by V. Shevchik in [25]. In particular, he proved that the asymptotic behaviour of the eigenvalues of $\mathcal{A}_{r}$ is "intermediate" between that one of the homogeneous boundary value problem for the Laplace operator on a domain $\left(\lambda_{k}(\Delta) \sim k\right)$ and the same problem for the biharmonic operator $\left(\lambda_{k}\left(\Delta^{2}\right) \sim k^{2}\right)$, namely $\lambda_{k}\left(\mathcal{A}_{r}\right) \sim k^{\frac{1}{3}}$.

Considering $\Gamma \subset \Omega$ a regular anisotropic $d$-set with respect to the anisotropy $a=$ $\left(\frac{4}{3}, \frac{2}{3}\right)$ and $t r^{\Gamma}$ the trace operator in the interpretation (1.3), the main objective of this work is to show that for sufficiently large $r$ the operator $\mathcal{A}_{r}^{-1}$ o $\operatorname{tr}^{\Gamma}$ is compact, non-negative, self-adjoint in the anisotropic Sobolev space

$$
\stackrel{\circ}{W}_{2}^{(1,2)}(\Omega)=\left\{u \in W_{2}^{(1,2)}(\Omega): u\left|\partial \Omega=\frac{\partial u}{\partial x_{2}}\right| \partial \Omega=0\right\}
$$

and that it is generated by the quadratic form $\int_{\Omega} f(\gamma) \overline{g(\gamma)} d \mu(\gamma)$ in $\dot{W}_{2}^{(1,2)}(\Omega)$. Moreover, we prove that there exist constants $c>0$ and $C>0$ such that its positive eigenvalues $\lambda_{k}\left(\mathcal{A}_{r}^{-1} \circ t r^{\Gamma}\right)$, repeated according to multiplicity and ordered by their magnitude, 
can be estimated by

$$
c k^{-\frac{1}{d}\left(d+\frac{2}{3}\right)} \leq \lambda_{k}\left(\mathcal{A}_{r}^{-1} \circ t r^{\Gamma}\right) \leq C k^{-\frac{1}{d}\left(d+\frac{2}{3}\right)} \quad(k \in \mathbb{N}) .
$$

The precise formulation of (1.6) is contained in Theorem 4.2.

To prove the second estimate in (1.6) we use mainly some mapping properties of the operator $\mathcal{A}_{r}$ which were proved by $\mathrm{H}$. Triebel in [28], some recent results from $[2,3]$ of $O$. V. Besov concerning interpolation of anisotropic function spaces and the inequality of $B$. Carl between the eigenvalues and the entropy numbers of a compact operator. The first estimate in (1.6) is proved using the atomic decomposition theorem in anisotropic function spaces and some Hilbert space techniques, in particular the theorem which states that for a compact, non-negative and self-adjoint operator acting in a Hilbert space its eigenvalues coincide with its approximation numbers.

Briefly about the organizing of the manuscript. Regular anisotropic $d$-sets are presented in Section 2 whereas the $L_{p}$-spaces on such fractals are presented in Section 3. The main result is formulated in Section 4 and it is proved in Section 5. Our aim was a self-contained work so that we included all necessary basic ingredients (entropy numbers, approximation numbers, atomic decompositions in anisotropic function spaces). The notation is standard. For a normed or quasi-normed space $X$ we denote by $\|x \mid X\|$ the norm of the vector $x$. The embedding of the quasi-normed space $X$ into the quasinormed space $Y$ is denoted $X \hookrightarrow Y$.

\section{Regular anisotropic fractals in $\mathbb{R}^{2}$}

Let $\Lambda=\left\{\Lambda_{1}, \ldots, \Lambda_{N}\right\}$ be a family of contractions of $\mathbb{R}^{2}$. It is well known (see [17] and cf. $[10,11]$ ) that there exists a unique (non-empty) compact set $\Gamma$ in $\mathbb{R}^{2}$ invariant with respect to $\Lambda$, i.e. such that $\Gamma=\Lambda_{1}(\Gamma) \cup \ldots \cup \Lambda_{n}(\Gamma)$. $\Gamma$ is called the fractal associated with the system $\Lambda$.

Let us assume that the contractions of the system $\Lambda$ are affinities, i.e. each $\Lambda_{i}$ has the form $\Lambda_{i}(x)=C_{i} x+h_{i}$ where $C_{i} \in \mathrm{GL}(2, \mathbb{R})$ and $h_{i} \in \mathbb{R}^{2}$. Let $\Gamma$ be the fractal associated with $\Lambda$. The real number $d=d_{\Lambda}(\Gamma)$ uniquely defined by

$$
\sum_{i=1}^{N}\left|\operatorname{det} C_{i}\right|^{\frac{d}{2}}=1
$$

is called the affine dimension of $\Gamma$ (see [32: Definition 4.12]). We want to remark that in the case of diagonal affinities the number $d_{\Lambda}(\Gamma)$ was already considered by $B$. Mandelbrot in [19] under the name gap dimension.

A particular case of affinities $\Lambda_{i}$ is of interest. Let $n_{1} \geq 2$ and $n_{2} \geq 2$ be integers and let $F$ be the subset of $\mathbb{R}^{2}$ given by

$$
F=\left\{\left(\frac{t_{1}}{n_{1}}, \frac{t_{2}}{n_{2}}\right): t_{1}, t_{2} \text { integers, } 0 \leq t_{1}<n_{1} \text { and } 0 \leq t_{2}<n_{2}\right\}
$$


Suppose that every element $\Lambda_{i}$ of the system $\Lambda$ is of the form $\Lambda_{i}(x)=C x+h_{i}$ where the translation vectors $h_{i}$ are in $F$ and

$$
C=\left(\begin{array}{cc}
\frac{1}{n_{1}} & 0 \\
0 & \frac{1}{n_{2}}
\end{array}\right) .
$$

Thus each $\Lambda_{i}$ maps the unit square $Q=[0,1] \times[0,1]$ onto a rectangle contained in $Q$. The fractal associated with $\Lambda$ is a generalisation of the Cantor set in the plane and is called general Sierpinski carpet in [20]. The Hausdorff dimension for this type of fractals was computed in [20] (see also [11: Example 9.11]). The definition of a a regular anisotropic fractal in [32: Subsection 4.18] is slightly more general. We present it below. Assume that every $\Lambda_{i}$ is of the form $\Lambda_{i}(x)=C_{i} x+h_{i}$ where

$$
C_{i}=\left(\begin{array}{cc}
\frac{ \pm 1}{n_{1}} & 0 \\
0 & \frac{ \pm 1}{n_{2}}
\end{array}\right)
$$

(the signs depend on $i$ and indicate a possible reflection) and the vectors $h_{i}$ still have as components integer multiples of $\frac{1}{n_{1}}$ and $\frac{1}{n_{2}}$ and are chosen such that $\Lambda_{i}(Q)$ is still contained in $Q$ and $\Lambda_{i}(Q)$ is disjoint from $\Lambda_{j}(Q)$ if $i \neq j$. The fractal generated by the affinities $\Lambda_{i}$ specified above is called a regular anisotropic fractal. In [32] the matrices $C_{i}$ are written in the form

$$
C_{i}=\left(\begin{array}{cc} 
\pm 2^{-\kappa a_{1}} & 0 \\
0 & \pm 2^{-\kappa a_{2}}
\end{array}\right)
$$

with

$$
a_{1}=\frac{2 \log n_{1}}{\log \left(n_{1} n_{2}\right)}, \quad a_{2}=\frac{2 \log n_{2}}{\log \left(n_{1} n_{2}\right)}, \quad \kappa=\frac{1}{2} \log \left(n_{1} n_{2}\right) .
$$

In particular, $a=\left(a_{1}, a_{2}\right)$ is a so-called two-dimensional anisotropy, that is $0<a_{2}, a_{1}<$ $\infty$ and $a_{1}+a_{2}=2$ (cf. the next section).

If $j \in \mathbb{N}_{0}$ and $N_{j} \in \mathbb{N}_{0}$, we deal with sets of open rectangles $\left\{R_{j l}: l=1, \ldots, N_{j}\right\}$ in $\mathbb{R}^{2}$ having sides parallel to the axes; the side length of the rectangle $R_{j l}$ with respect to the $x_{i}$-axis is denoted by $r_{i}^{j, l}$ where $i=1,2$. We will always assume that the side lengths of the rectangles $R_{j l}$ are ordered in the same way, for example $r_{1}^{j, l} \leq r_{2}^{j, l}$ for any $j \in \mathbb{N}_{0}$ and any $l=1, \ldots, N_{j}$.

Theorem 2.1 [13: Theorem 3.1]. Let $\Gamma$ be the fractal generated by the system $\Lambda$ of affinities specified by $(2.2)-(2.4)$, having affine dimension $d=d_{\Lambda}(\Gamma)$ according to (2.1). There exist numbers $c_{1}, c_{2}>0$ such that for any $j \in \mathbb{N}_{0}$ : there exists a natural number $N_{j}$ with

$$
c_{1} 2^{j d} \leq N_{j} \leq c_{2} 2^{j d} \quad \text { if } j \in \mathbb{N}, N_{0}=1,
$$

and there exists a finite sequence of open rectangles $\left\{R_{j l}: l=1, \ldots, N_{j}\right\}$ having sides parallel to the axes, $R_{01}=\dot{Q}$, satisfying the following conditions:

(i) There exists a constant $0 .<c_{0} \leq 1$ such that for all $j \in \mathbb{N}_{0}$ and all $l=1, \ldots, N_{j}$

$$
\left(c_{0} 2^{-j}\right)^{a_{i}} \leq r_{i}^{j l} \leq 2^{-j a_{i}} \quad(i=1,2) .
$$



$R_{j l}$.

(ii) If $l \neq l^{\prime}$, then $R_{j l} \cap R_{j l^{\prime}}=\emptyset$.

(iii) For any rectangle $R_{j+1, k}$ there is a rectangle $R_{j l}, l=l(k)$, such that $R_{j+1, k} \subset$

(iv) For any $j \in \mathbb{N}_{0}$ and any $l=1, \ldots, N_{j},\left(\operatorname{vol} R_{j l}\right)^{\frac{d}{2}}=\sum_{R_{j+1, k} \subset R_{j l}}\left(\operatorname{vol} R_{j+1, k}\right)^{\frac{d}{2}}$.

(v) $\Gamma=\bigcap_{j=0}^{\infty} \bigcup_{l=1}^{N_{j}} \overline{R_{j l}}$.

Taking into account Theorem 2.1 and the terminology from [32: Subsection 4.18] the fractal $\Gamma$ is called a regular anisotropic $d$-set with respect to the anisotropy $a=\left(a_{1}, a_{2}\right)$ (see [13]).

As remarked in [32: Subsections 4.14 and 4.22], if the anisotropy $a=\left(a_{1}, a_{2}\right)$ is non-trivial, that means $a=\left(a_{1}, a_{2}\right) \neq(1,1)$, then the number $d$ from (2.1) and the Hausdorff dimension $\operatorname{dim}_{H} \Gamma$ of the fractal $\Gamma$, are completely unrelated. Furthermore, it can happen that the regular anisotropic $d$-set $\Gamma$ is also an isotropic $d^{\prime}$-set with $d \neq d^{\prime}$ (see [32: Subsection 4.21]).

For a detailed discussion concerning dimension problems for self-affine fractals the reader is referred to the recent work [1].

Theorem 2.2 [32: Theorems 5.5 and 4.15]. Let $\Gamma$ be a regular anisotropic d-set with respect to the anisotropy $a=\left(a_{1}, a_{2}\right)$ and let $\left\{R_{j l}: j \in \mathbb{N}_{0}, l=1, \ldots, N_{j}\right\}$ be the rectangles from Theorem 2.1. Then there exists a Radon measure $\mu$ in $\mathbb{R}^{2}$ uniquely determined with supp $\mu=\Gamma$ and

$$
\mu\left(\Gamma \cap R_{j l}\right)=\left(\operatorname{vol} R_{j l}\right)^{\frac{d}{2}} \quad \text { for all } j \in \mathbb{N}_{0} \text { and } l=1, \ldots, N_{j} .
$$

As it was remarked by M. Bricchi (cf. [5]), following the proofs in [32: Theorems 5.5 and 4.15] and [17] it is not difficult to see that the measure $\mu$ from Theorem 2.2 coincides with the unique Borel regular (outer) measure $\mu$ constructed in [17], with supp $\mu=\Gamma$ and of total mass 1 which is invariant with respect to $\Lambda$, that is

$$
\mu(E)=\frac{1}{N} \sum_{i=1}^{N} \mu\left(\Lambda_{i}^{-1}(E)\right) \quad \text { for all } E \subset \mathbb{R}^{2}
$$

We know that if $\Gamma$ is an isotropic $d$-set with underlying measure $\mu$ and if $0<\kappa<1$, then

$$
\mu(B(\gamma, \kappa r) \cap \Gamma) \sim \mu(B(\gamma, r) \cap \Gamma) \sim r^{d}
$$

where the equivalence constants depend on $\kappa$ but not on $\gamma \in \Gamma$ and $0 \dot{<} r \leq 1$. For a regular anisotropic $d$-set $\Gamma$ we have (2.5) but no counterpart of (2.6). At least a weak version of (2.6) will be needed. If $0<\kappa<1$, then $\kappa R_{j l}$ denotes the rectangle concentric with $R_{j l}$ and with side lengths respectively $\kappa r_{1}^{j, l}$ and $\kappa r_{2}^{j, l}$.

Definition 2.3. A regular anisotropic $d$-set with respect to the anisotropy $a=$ $\left(a_{1}, a_{2}\right)$ equipped with measure $\mu$ according to Theorem 2.2 is called proper if there exist two numbers $0<\kappa<1$ and $0<c \leq 1$ such that

$$
\mu\left(\Gamma \cap \kappa R_{j l}\right) \geq c\left(\operatorname{vol} R_{j l}\right)^{\frac{d}{2}} \quad\left(j \in \mathbb{N}_{0}, l=1, \ldots, N_{j}\right)
$$


where $\left\{R_{j l}: j \in \mathbb{N}_{0}\right.$ and $\left.l=1, \ldots, N_{j}\right\}$ are the rectangles from Theorem 2.1.

This is a slightly different version of [32: Definition 5.11]. Since $\Gamma$ is generated by linear contractions, following the lines of the proof of [32: Proposition 5.13] it turns out that if $\Gamma \cap \dot{Q} \neq \emptyset$, then $\Gamma$ is proper. Hence condition (2.7) is rather natural.

\section{3. $L_{p}$-spaces on regular anisotropic fractals}

3.1 Some preliminaries on anisotropic function spaces. If $1<p<\infty$ and $\left(s_{1}, s_{2}\right)$ is a pair of natural numbers, then the classical anisotropic Sobolev space $W_{p}^{\left(s_{1}, s_{2}\right)}\left(\mathbb{R}^{2}\right)$ is the collection of all tempered distributions $f \in S^{\prime}\left(\mathbb{R}^{2}\right)$ such that

$$
\left\|f\left|W_{p}^{\left(s_{1}, s_{2}\right)}\left(\mathbb{R}^{2}\right)\|=\| f\right| L_{p}\left(\mathbb{R}^{2}\right)\right\|+\left\|\frac{\partial^{s_{1}} f}{\partial x_{1}^{s_{1}}}\left|L_{p}\left(\mathbb{R}^{2}\right)\|+\| \frac{\partial^{s_{2}} f}{\partial x_{2}^{s_{2}}}\right| L_{p}\left(\mathbb{R}^{2}\right)\right\|
$$

is finite. In contrast to the usual (isotropic) Sobolev space $\left(s_{1}=s_{2}\right)$ the smoothness properties of an element from $W_{p}^{\left(s_{1}, s_{2}\right)}\left(\mathbb{R}^{2}\right)$ depend on the chosen direction in $\mathbb{R}^{2}$. The number $s$ defined by

$$
\frac{1}{s}=\frac{1}{2}\left(\frac{1}{s_{1}}+\frac{1}{s_{2}}\right)
$$

is usually called the "mean smoothness" and $a=\left(a_{1}, a_{2}\right)$, where $a_{1}=\frac{s}{s_{1}}$ and $a_{2}=\frac{s}{s_{2}}$, characterises the anisotropy. Sometimes we will use the notation $W_{p}^{s, a}\left(\mathbb{R}^{2}\right)$ for these spaces.

We recall now the definition of anisotropic Besov spaces on $\mathbb{R}^{2}$. First we fix some notation. A pair $a=\left(a_{1}, a_{2}\right)$ with $0<a_{1}, a_{2}<\infty$ and $a_{1}+a_{2}=2$ is called an anisotropy. If $a=(1,1)$, then we speak about the "isotropic case". The action of $t \in[0, \infty)$ on $x=\left(x_{1}, x_{2}\right) \in \mathbb{R}^{2}$ is defined by the formula

$$
t^{a} x=\left(t^{a_{1}} x_{1}, t^{a_{2}} x_{2}\right)
$$

For $t>0$ and $s \in \mathbb{R}$ let $t^{s a} x=\left(t^{s}\right)^{a} x$. In particular, $t^{-a} x=\left(t^{-1}\right)^{a} x$ and $2^{-j a} x=$ $\left(2^{-j}\right)^{a} x$.

For $x=\left(x_{1}, x_{2}\right) \in \mathbb{R}^{2}$ and $x \neq 0$ let $|x|_{a}$ be the unique positive number $t$ such that

$$
\frac{x_{1}^{2}}{t^{2 a_{1}}}+\frac{x_{2}^{2}}{t^{2 a_{2}}}=1
$$

and let $|0|_{a}=0$. By M. Yamazaki [34: Theorem $\left.1.4 / 3,8\right],|\cdot|_{a}$ is an anisotropic distance function in $C^{\infty}\left(\mathbb{R}^{2} \backslash\{0\}\right)$. Remark that in the isotropic case $|x|_{a}$ is the Euclidean distance of $x$ to the origin.

Let $\varphi_{0}$ a $C^{\infty}$ function on $\mathbb{R}^{2}, \varphi_{0}(x)=1$ if $|x|_{a} \leq 1, \operatorname{supp} \varphi_{0} \subset\left\{x \in \mathbb{R}^{2}:|x|_{a} \leq 2\right\}$ and $\varphi_{j}(x)=\varphi_{0}\left(2^{-j a} x\right)-\varphi_{0}\left(2^{(-j+1) a} x\right)$ if $j \in \mathbb{N}$. Then $\sum_{j=0}^{\infty} \varphi_{j}(x)=1$ if $x \in \mathbb{R}^{2}$ and $(\varphi j)_{j \in N_{0}}$ is a smooth anisotropic dyadic resolution of unity (cf. [24: Section 4.2]). 
Let $0<p \leq \infty, 0<q \leq \infty$ and $s \in \mathbb{R}$. The anisotropic Besov space $B_{p q}^{s, a}\left(\mathbb{R}^{2}\right)$ consists of all tempered distributions $f \in S^{\prime}\left(\mathbb{R}^{2}\right)$ for which the quasi-norm

$$
\left\|f \mid B_{p q}^{s, a}\left(\mathbb{R}^{2}\right)\right\|=\left(\sum_{j=0}^{\infty} 2^{j, q}\left\|\left(\varphi_{j} \hat{f}\right)^{\vee} \mid L_{p}\left(\mathbb{R}^{2}\right)\right\|^{q}\right)^{\frac{1}{q}}
$$

(with usual modification if $q=\infty$ ) is finite. Here $\hat{f}$ and $g^{\vee}$ denote the Fourier transform of $f$ respectively the inverse Fourier transform of $g$. These are quasi-Banach spaces (Banach spaces if $p \geq 1$ and $q \geq 1$ ) which are independent of the choice of $\left(\varphi_{j}\right)_{j \in \mathbf{N}_{0}}$. The space $H_{2}^{s, a}\left(\mathbb{R}^{2}\right)=B_{22}^{s, a}\left(\mathbb{R}^{2}\right)$ is the anisotropic fractional Sobolev space. In particular, if $s_{1}=\frac{s}{a_{1}}$ and $s_{2}=\frac{s}{a_{2}}$ are natural numbers, then $B_{22}^{s, a}\left(\mathbb{R}^{2}\right)=W_{2}^{s, a}\left(\mathbb{R}^{2}\right)$.

Directly from the definition we have

$$
B_{p q_{0}}^{s+e, a}\left(\mathbb{R}^{2}\right) \hookrightarrow B_{p q_{1}}^{s, a}\left(\mathbb{R}^{2}\right) \quad \text { if } \varepsilon>0
$$

for any $s \in \mathbb{R}$, any $0<p \leq \infty$, and any $0<q_{0}, q_{1} \leq \infty$ (see [27: Proposition 2.3.2/2]). Anisotropic function spaces of $F_{p q}^{s, a}\left(\mathbb{R}^{2}\right)$ type, $0<p<\infty$, are defined changing the roles of the spaces $L_{p}\left(\mathbb{R}^{2}\right)$ and $l_{q}$ in the above definition but we do not stress this point here. The above spaces are denoted $B_{p q}^{s}\left(\mathbb{R}^{2}\right)$ in the isotropic case. A systematic treatment of the theory of isotropic $B_{p q}^{s}\left(\mathbb{R}^{2}\right)$ and $F_{p q}^{s}\left(\mathbb{R}^{2}\right)$ spaces may be found in the books of $\mathrm{H}$. Triebel $[27,30]$.

Anisotropic function spaces have been studied in great detail by S. M. Nikol'skij (see [23]) and by O. V. Besov, V. P. Il'in and S. M. Nikol'skij (see [4]). For a list of contributions to the theory of anisotropic function spaces we refer to [12].

If $s \in \mathbb{R}, 1<p<\infty, 1 \leq q \leq \infty$, and $\Omega \subset \mathbb{R}^{2}$ is a domain, then $B_{p q}^{s, a}(\Omega)$ is the restriction of $B_{p q}^{s, a}\left(\mathbb{R}^{2}\right)$ to $\Omega$ normed by

$$
\left\|f\left|B_{p q}^{s, a}(\Omega)\|=\inf \| g\right| B_{p q}^{s, a}\left(\mathbb{R}^{2}\right)\right\|
$$

where the infimum is taken over all $g \in B_{p q}^{s, a}\left(\mathbb{R}^{2}\right)$ with $g|\Omega=f| \Omega$ (in the sense of distributions on $\Omega$ ). Of course, this definition works for all (bounded or unbounded) domains $\Omega$ but we will be interested later only on the unit disc. Let us recall that

$$
W_{2}^{s, a}(\Omega)=B_{22}^{s, a}(\Omega) \quad \text { if } s>0 .
$$

For $s \in \mathbb{R}, 1<p<\infty$, and $1 \leq q \leq \infty$ we will use the (non-standard) notation

$$
\dot{B}_{p q}^{s, a}(\Omega)=\left\{f \in B_{p q}^{s, a}(\Omega): f\left|\partial \Omega=\frac{\partial f}{\partial x_{2}}\right| \partial \Omega=0\right\} .
$$

3.2 Embeddings of $L_{p}(\Gamma)$ in anisotropic Besov spaces. Let $\Gamma$ be a regular anisotropic $d$-set with respect to the given anisotropy $a=\left(a_{1}, a_{2}\right)$. The $L_{p}$-spaces on $\Gamma(0<p \leq \infty)$ are introduced in the usual way with respect to the underlying Radon measure $\mu$ on $\Gamma$ according to Theorem 2.2. If $1 \leq p \leq \infty$ any $f_{\Gamma} \in L_{p}(\Gamma)$ will be interpreted as a tempered distribution $f \in S^{\prime}\left(\mathbb{R}^{2}\right)$ given by

$$
f(\varphi)=\int_{\Gamma} f_{\Gamma}(\gamma)(\varphi \mid \Gamma)(\gamma) d \mu(\gamma) \quad\left(\varphi \in S\left(\mathbb{R}^{2}\right)\right),
$$

where $\varphi \mid \Gamma$ is the restriction of $\varphi$ to $\Gamma$. 
Theorem 3.1. Let $0<d<2$ and let $\Gamma$ be a regular anisotropic $d$-set in $\mathbb{R}^{2}$ with respect to the anisotropy $a=\left(a_{1}, a_{2}\right)$. If $1<p \leq \infty$ and $\frac{1}{p}+\frac{1}{p^{\prime}}=1$, then (in the sense of (3.6))

$$
L_{p}(\Gamma)=\left\{f \in B_{p \infty}^{-\frac{2-d}{p^{\prime}}, a}\left(\mathbb{R}^{2}\right): f(\varphi)=0 \text { if } \varphi \in S\left(\mathbb{R}^{2}\right) \text { and } \varphi \mid \Gamma=0\right\} \text {. }
$$

The above theorem was proved in [13: Theorem 3.6] and it is the anisotropic counterpart of [33: Theorem 2] (see also [32: Theorem 18.2]).

3.3 Traces of anisotropic Besov spaces on fractals. Assume that $\Gamma$ is a regular anisotropic $d$-set in $\mathbb{R}^{2}$ with respect to the anisotropy $a=\left(a_{1}, a_{2}\right)$. If $\varphi \in S\left(\mathbb{R}^{2}\right)$, then $\operatorname{tr}_{\Gamma} \varphi=\varphi \mid \Gamma$ makes sense pointwise. If $0<p, q<\infty$ and $s \in \mathbb{R}$, then the embedding $\operatorname{tr}_{\Gamma} B_{p q}^{s, a}\left(\mathbb{R}^{2}\right) \hookrightarrow L_{p}(\Gamma)$ must be understood as follows: there exists a positive number $c>0$ such that, for any $\varphi \in S\left(\mathbb{R}^{2}\right),\left\|\operatorname{tr}_{\Gamma} \varphi\left|L_{p}(\Gamma)\|\leq c\| \varphi\right| B_{p q}^{\text {sa }}\left(\mathbb{R}^{2}\right)\right\|$. Since $S\left(\mathbb{R}^{2}\right)$ is dense in $B_{p q}^{s, a}\left(\mathbb{R}^{2}\right)$ for $0<p, q<\infty$ this inequality can be extended by completion to any $f \in B_{p q}^{s, a}\left(\mathbb{R}^{2}\right)$ and the resulting function is denoted $\operatorname{tr}_{\Gamma} f$. In addition, the equality $\operatorname{tr}_{\Gamma} B_{p q}^{s, a}\left(\mathbb{R}^{2}\right)=L_{p}(\Gamma)$ means that any $f_{\Gamma} \in L_{p}(\Gamma)$ is the trace of a suitable $g \in B_{p q}^{s, a}\left(\mathbb{R}^{2}\right)$ on $\Gamma$ and $\left\|f_{\Gamma} \mid L_{p}(\Gamma)\right\| \sim \inf \left\{\left\|g \mid B_{p q}^{s, a}\left(\mathbb{R}^{2}\right)\right\|: \operatorname{tr}_{\Gamma} g=f_{\Gamma}\right\}$.

Theorem 3.2. Let $0<d<2$ and let $\Gamma$ be a regular anisotropic d-set in $\mathbb{R}^{2}$ with respect to the anisotropy $a=\left(a_{1}, a_{2}\right)$. If $\frac{d}{2}<p<\infty$ and $0<q \leq \min (1, p)$, then

$$
\operatorname{tr}_{\Gamma} B_{p q}^{\frac{2-d}{p}, a}\left(\mathbb{R}^{2}\right)=L_{p}(\Gamma) .
$$

The above theorem was proved in [13: Theorem 3.7] and it is the anisotropic counterpart of [33: Theorem 3] (see also [32: Theorem 18.6]).

\section{The main result}

Let $\Omega=\left\{x=\left(x_{1}, x_{2}\right) \in \mathbb{R}^{2}: x_{1}^{2}+x_{2}^{2}<1\right\}$ be the unit disc in the plane and let $\partial \Omega$ be its boundary. In what follows we will consider $\Gamma \subset \Omega$ a regular anisotropic $d$-set with respect to the anisotropy $a=\left(\frac{4}{3}, \frac{2}{3}\right)$. One can take, for example, $n_{1}=16$ and $n_{2}=4$ in (2.4) and (2.2). In the sequel we shall not distinguish between $f_{\Gamma}$ as an element of some $L_{p}(\Gamma)$ and as the distribution belonging to some $B_{p \infty}^{-s, a}(\Omega)$ according to (3.7).

To avoid any misunderstanding we emphasise that the trace operator has two different meanings which we distinguish by $t r_{\Gamma}$ and $t r^{r}$ if extra clarity is desirable. If $1<p<\infty$, then by $(3.8)$

$$
\operatorname{tr}_{\Gamma}: B_{p 1}^{\frac{2-d}{p}, a}(\Omega) \rightarrow L_{p}(\Gamma)
$$

and if one applies in addition (3.7)

$$
\operatorname{tr} \Gamma: B_{p 1}^{\frac{2-d}{p}, a}(\Omega) \rightarrow B_{p \infty}^{-\frac{2-d}{p^{\prime}}, a}(\Omega)
$$

The latter can be rephrased asking for an optimal extension of $\operatorname{tr}^{\Gamma}$ considered as a mapping from $D(\Omega)$ into $D^{\prime}(\Omega)$ given by (1.3) (cf. [32: Subsection 28.1]). 
We consider the anisotropic Sobolev space

$$
W_{2}^{(1,2)}(\Omega)=\left\{u \in S^{\prime}(\Omega):\left\|u\left|L_{2}(\Omega)\|+\| \frac{\partial u}{\partial x_{1}}\right| L_{2}(\Omega)\right\|+\left\|\frac{\partial^{2} u}{\partial x_{2}^{2}} \mid L_{2}(\Omega)\right\|<\infty\right\} .
$$

Clearly (see (3.1)), the mean smoothness is $s=\frac{4}{3}$ and $a=\left(\frac{4}{3}, \frac{2}{3}\right)$ characterises the anisotropy. Using the notation from the previous section we have

$$
W_{2}^{\frac{4}{3}, a}(\Omega)=W_{2}^{(1,2)}(\Omega) .
$$

Remark 4.1. Let

$$
\stackrel{\circ}{W}_{2}^{(1,2)}(\Omega)=\left\{u \in W_{2}^{(1,2)}(\Omega): u\left|\partial \Omega=\frac{\partial u}{\partial x_{2}}\right| \partial \Omega=0\right\}
$$

and let $\mathcal{A}_{r}$ be the operator defined in (1.5). We know from [28: Proposition 1] that there exists an $r_{0} \in \mathbb{R}$ such that for any $r>r_{0}$ the operator $\mathcal{A}_{r}^{\frac{1}{2}} \operatorname{maps} \dot{W}_{2}^{(1,2)}(\Omega)$ isomorphically onto $L_{2}(\Omega)$; consequently, we may fix the norm on $W_{2}^{(1,2)}(\Omega)$ by

$$
\left\|u\left|\dot{W}_{2}^{(1,2)}(\Omega)\|=\| \mathcal{A}_{r}^{\frac{1}{2}} u\right| L_{2}(\Omega)\right\|
$$

and a corresponding scalar product.

We are able now to present our main result.

Theorem 4.2. Let $0<d<2$ and let $\Omega$ be the unit disc in the plane. Let $\Gamma \subset \Omega$ be a regular anisotropic d-set with respect to the anisotropy $a=\left(\frac{4}{3}, \frac{2}{3}\right)$. Let $t^{\Gamma}$ be the trace operator in the interpretation (4.2) and (1.3) whereas $\operatorname{tr}_{\Gamma}$ stands for the trace operator according to (4.1). Let $\mathcal{A}_{r}$ be the operator from (1.5).

(i) There exists $r_{0} \in \mathbb{R}$ such that for any $r>r_{0}$ the operator

$$
T=\mathcal{A}_{r}^{-1} \circ \operatorname{tr}^{\Gamma}
$$

is compact, non-negative, self-adjoint in $\dot{W}_{2}^{(1,2)}(\Omega)$ and has null space

$$
N(T)=\left\{f \in \dot{W}_{2}^{(1,2)}(\Omega): \operatorname{tr}_{\Gamma} f=0\right\} .
$$

Furthermore, $T$ is generated by the quadratic form in $\dot{W}_{2}^{(1,2)}$

$$
\int_{\Gamma} f(\gamma) \overline{g(\gamma)} d \mu(\gamma)=(T f, g)_{W_{2}^{(1,2)}(\Omega)} \text { where } f, g \in \stackrel{\circ}{W}_{2}^{(1,2)}(\Omega)
$$

and $\mu$ is the Radon measure according to Theorem 2.2 .

(ii) Let $r>r_{0}$. There exists a constant $C>0$ such that positive eigenvalues $\lambda_{k}(T)$ of $T$, repeated according to the multiplicity and ordered by their magnitude, can be estimated by

$$
\lambda_{k}(T) \leq C k^{-\frac{1}{d}\left(d+\frac{2}{3}\right)} \quad(k \in \mathbb{N}) .
$$

If in addition, $\Gamma$ is proper according to Definition 2.3 , then there exists a constant $c>0$ such that

$$
c k^{-\frac{1}{d}\left(d+\frac{2}{3}\right)} \leq \lambda_{k}(T) \quad(k \in \mathbb{N}) .
$$

The detailed proof is given in the next section.

Remark 4.3. The additional assumption on $\Gamma$ to be proper in the estimate (4.8) excludes by the considerations after Definition 2.3 only pathological cases where the whole fractal retreats in the boundary of the starting square. 


\section{Proof of Theorem 4.2}

Proof of Theorem 4.2/(i). Let $a=\left(\frac{4}{3}, \frac{2}{3}\right)$. Using the elementary embedding $W_{2}^{(1,2)}(\Omega)=W_{2}^{\frac{1}{3}, a}(\Omega) \hookrightarrow B_{22}^{\frac{2-d}{2}, a}(\Omega)$ (see (3.2)) and applying (3.8) there exists a constant $c>0$ such that

$$
\left\|\operatorname{tr}_{\Gamma} f\left|L_{2}(\Gamma)\|\leq c\| f\right| \stackrel{\mathscr{W}}{2}_{2}^{(1,2)}(\Omega)\right\| \quad \text { for any } f \in \dot{W}_{2}^{(1,2)}(\Omega) .
$$

Defining

$$
q(f, g)=\int_{\Gamma} f(\gamma) \overline{g(\gamma)} d \mu(\gamma) \quad \text { for any } f, g \in \stackrel{\mathscr{W}}{2}^{(1,2)}(\Omega)
$$

it is clear that $q(\cdot, \cdot)$ is a non-negative quadratic form in $\dot{W}_{2}^{(1,2)}(\Omega)$. By [31: page 91] there exists a non-negative and self-adjoint operator $T$ uniquely determined such that

$$
q(f, g)=(T f, g)_{W_{2}^{(1,2)}(\Omega)} \quad \text { for any } f, g \in \stackrel{\circ}{2}_{2}^{(1,2)}(\Omega)
$$

Furthermore,

$$
\left\|\operatorname{tr}_{\Gamma} f\left|L_{2}(\Gamma)\|=\| \sqrt{T} f\right| \dot{W}_{2}^{(1,2)}(\Omega)\right\|
$$

where $\sqrt{T}=T^{\frac{1}{2}}$ and this proves (4.5).

So it remains to prove that the above operator is the same as in (4.4). Let $f \in$ $\mathscr{W}_{2}^{(1,2)}(\Omega)$ and $\varphi \in D(\Omega)$. Then

$$
\int_{\Gamma} f(\gamma) \overline{\varphi(\gamma)} d \mu(\gamma)=(T f, \varphi)_{W_{2}^{(1,2)}(\Omega)}=\left(\mathcal{A}_{r}^{\frac{1}{2}} T f, \mathcal{A}_{r}^{\frac{1}{2}} \varphi\right)_{L_{2}(\Omega)}=\left(\mathcal{A}_{r} T f, \varphi\right)_{L_{2}(\Omega)}
$$

the second equality in (5.3) being justified by the fact that for $r>r_{0}$ we fixed the norm in $\grave{W}_{2}^{(1,2)}(\Omega)$ by $\left\|\mathcal{A}_{r}^{\frac{1}{2}}(\cdot) \mid L_{2}(\Omega)\right\|$ and a corresponding scalar product (see Remark 4.1). Considered as a dual pairing in $\left(D(\Omega), D^{\prime}(\Omega)\right)$ we obtain $\mathcal{A}_{r} T f=\operatorname{tr}^{\Gamma} f$ and (4.6) follows by the same arguments as in [32: Theorem 27.15/Step 1]. This completes the first part of the proof of Theorem 4.2.

5.2 Proof of the estimate (4.7). Step 1: Basic facts on entropy numbers and their relation to the eigenvalues. Let $B_{1}$ and $B_{2}$ two quasi-Banach spaces. The family of all linear bounded operators $U: B_{1} \rightarrow B_{2}$ is denoted by $L\left(B_{1}, B_{2}\right)$ or $L\left(B_{1}\right)$ if $B_{1}=B_{2}$. We will assume that the reader is familiar with the definition of the entropy numbers $e_{k}(U)(k \in \mathbb{N})$ of a compact map $U \in L\left(B_{1}, B_{2}\right)$. This definition is given, for example, in [8: Subsection 1.3.1] where one can find also comments and historical references. We only want to mention here that if $B_{1}, B_{2}, B_{3}$ are quasi-Banach spaces, if $X \in L\left(B_{1}, B_{2}\right)$ and $R \in L\left(B_{2}, B_{3}\right)$, then for all $k, l \in \mathbb{N}$,

$$
e_{k+l-1}(R \circ X) \leq e_{k}(R) e_{l}(X)
$$

(see [8: Lemma 1.3.1/1]).

Let $B$ be a complex quasi-Banach space and $U \in L(B)$ a compact map. We know from [8: Theorem 1.2] that the spectrum of $U$, apart from the point 0 , consists solely 
of eigenvalues of finite algebraic multiplicity: let $\left\{\lambda_{k}(U): k \in \mathbb{N}\right\}$ be the sequence of all non-zero eigenvalues of $U$, repeated according to algebraic multiplicity and ordered so that

$$
\left|\lambda_{1}(U)\right| \geq\left|\lambda_{2}(U)\right| \geq \ldots \geq 0 .
$$

If the operator $U$ has only $m \quad(<\infty)$ distinct eigenvalues and $M$ is the sum of their algebraic multiplicities, we put $\lambda_{k}(U)=0$ for $k>M$. Due to B. Carl (see [6]) we know the following connection between the eigenvalues of the operator $U$ and its entropy numbers: Let $U$ and $\left\{\lambda_{k}(U): k \in \mathbb{N}\right\}$ as above. Then

$$
\left|\lambda_{k}(U)\right| \leq \sqrt{2} e_{k}(U)
$$

Step 2: Entropy numbers for traces on regular anisotropic $d$-sets.

Theorem 5.1. Let $0<d<2$ and let $\Gamma$ be a regular anisotropic $d$-set in $\mathbb{R}^{2}$ with respect to the anisotropy $a=\left(a_{1}, a_{2}\right)$. Let $0<p_{1} \leq \infty, 0<p_{2} \leq \infty, 0<q \leq \infty$ and $s \in \mathbb{R}$ such that

$$
\delta_{+}=s-d\left(\frac{1}{p_{1}}-\frac{1}{p_{2}}\right)_{+}>0 .
$$

Then the trace operator

$$
\operatorname{tr}_{\Gamma}: B_{p_{1} q}^{s+\frac{2-d}{p_{1}}, a}\left(\mathbb{R}^{2}\right) \rightarrow L_{p_{2}}(\Gamma)
$$

is compact and there exists a constant $C>0$ such that, for all $k \in \mathbb{N}$,

$$
e_{k}\left(\operatorname{tr}_{\Gamma}: B_{p_{1} q}^{s+\frac{2-d}{p_{1}}, a}\left(\mathbb{R}^{2}\right) \rightarrow L_{p_{2}}(\Gamma)\right) \leq C k^{-\frac{1}{d}}
$$

The above theorem was proved in [13: Theorem 6.1] and it is the anisotropic counterpart of [32: Theorem 20.6] (cf. also [32: Theorem 22.2]). If, in addition, $\Gamma$ is proper according to Definition 2.3 , then there exists a constant $c>0$ such that, for all $k \in \mathbb{N}$,

$$
c k^{-\frac{\dot{g}}{d}} \leq e_{k}\left(\operatorname{tr}_{\Gamma}: B_{p_{1} q}^{s+\frac{2-d}{p_{1}}, a}\left(\mathbb{R}^{2}\right) \rightarrow L_{p_{2}}(\Gamma)\right)
$$

But the last estimate will not be used later. We want to remark also that assumption (5.7) is crucial for the compactness of the operator in (5.8) and that this assumption cannot be weakened by $\delta_{+}=0$ (see [32: Subsection 20.7]).

Step 3: Let $a=\left(\frac{4}{3}, \frac{2}{3}\right)$ and let, according to $(4.3), \dot{W}_{2}^{\frac{4}{3}, a}(\Omega)=\dot{W}_{2}^{(1,2)}(\Omega)$. Let also $r>r_{0}$ and recall the notation (3.5). We prove now that the operator $T=\mathcal{A}_{r}^{-1} \circ t^{\Gamma}$ can be factorised by

$$
T=i d_{2} \circ \mathcal{A}_{r}^{-1} \circ i d_{1} \circ \operatorname{tr}_{\Gamma}
$$

where

$$
\begin{aligned}
\operatorname{tr}_{\Gamma} & : \dot{W}_{2}^{\frac{4}{3}, a}(\Omega) \rightarrow L_{2}(\Gamma) \\
i d_{1} & : L_{2}(\Gamma) \rightarrow B_{2 \infty}^{-\frac{2-d}{2}, a}(\Omega) \\
\mathcal{A}_{r}^{-1} & : B_{2 \infty}^{-\frac{2-d}{2}, a}(\Omega) \rightarrow \dot{B}_{2 \infty}^{\frac{8}{3}-\frac{2-d}{2}, a}(\Omega) \\
i d_{2} & : \dot{B}_{2 \infty}^{\frac{8}{3}-\frac{2-d}{2}, a}(\Omega) \rightarrow \dot{W}_{2}^{\frac{4}{3}, a}(\Omega) .
\end{aligned}
$$


The boundedness of $\operatorname{tr}_{\Gamma}$ in the first line of (5.9) was discussed in the previous section (see (5.1)). According to (3.3) the embedding $i d_{1}$ is (3.7) whereas the embedding $i d_{2}$ is a simple consequence of the inequality $\frac{8}{3}-\frac{2-d}{2}>\frac{4}{3}$ and of the elementary embedding (3.2) between anisotropic function spaces.

So it remains only to justify the boundedness of $\mathcal{A}_{r}^{-1}$ as indicated in the third line of (5.9). Let us denote $x^{0}=(-1,0)$ and $x^{1}=(1,0)$ and let $W_{2}^{\frac{8}{3}, a}\left(\Omega, x^{0}, x^{1}\right)=$ $W_{2}^{(2,4)}\left(\Omega, x^{0}, x^{1}\right)$ be the collection of all $f \in W_{2}^{\frac{8}{3}, a}(\Omega)$ such that

$$
\frac{\partial^{m_{1}+m_{2}} f}{\partial x_{1}^{m_{1}} \partial x_{2}^{m_{2}}}\left(x^{0}\right)=\frac{\partial^{m_{1}+m_{2}} f}{\partial x_{1}^{m_{1}} \partial x_{2}^{m_{2}}}\left(x^{1}\right)=0 \quad \text { if } 2 m_{1}+m_{2}+\frac{3}{2}<4
$$

(see [28: Formula (17)]). Let also

$$
\dot{W}_{2}^{\frac{\mathrm{a}}{3}, a}\left(\Omega, x^{0}, x^{1}\right)=\left\{f \in W_{2}^{\frac{8}{3}, a}\left(\Omega, x^{0}, x^{1}\right): f\left|\partial \Omega=\frac{\partial f}{\partial x_{2}}\right| \partial \Omega=0\right\} .
$$

Recall that there exists a number $r_{0} \in \mathbb{R}$ such that for any $r>r_{0}$ the operator

$$
\mathcal{A}_{\text {r maps }} \dot{W}_{2}^{\frac{6}{3}, a}\left(\Omega, x^{0}, x^{1}\right) \text { isomorphically onto } L_{2}(\Omega)
$$

(this was proved by H. Triebel in [28: Theorem 4]; see also [25: Theorem 2.1]). Furthermore, by [28: Proposition 2 and Remark 6] the operator

$$
\mathcal{A}_{r} \text { maps } \dot{W}_{2}^{\frac{4}{3}, a}(\Omega) \text { isomorphically onto } B_{22}^{-\frac{4}{3}, a}(\Omega) \text {. }
$$

Let

$$
\theta=\frac{3(2-d)}{8}
$$

Then, clearly, we have $0<\theta<1$ and $(1-\theta) \cdot 0+\theta \cdot\left(-\frac{4}{3}\right)=-\frac{2-d}{2}$. Since the unit disc $\Omega$ is a domain in $\mathbb{R}^{2}$ having the so-called $C^{m}$ flexible $\left(\frac{4}{3}, \frac{2}{3}\right)$ horn condition for any $m \in \mathbb{N}_{0}^{2}$ (see [2: Definition 1.2]), by [3] with the above $\theta$ we have the interpolation result

$$
\left(L_{2}(\Omega), B_{22}^{-\frac{4}{3}, a}(\Omega)\right)_{\theta, \infty}=B_{2 \infty}^{-\frac{2-d}{2}, a}(\Omega) .
$$

From (5.11), (5.12) and (5.14) it follows that for $r>r_{0}$ sufficiently large the operator

$$
\mathcal{A}_{r}^{-1} \text { maps } B_{2 \infty}^{-\frac{2-d}{2}, a}(\Omega) \text { bounded into }\left(\dot{W}_{2}^{\frac{8}{3}, a}\left(\Omega, x^{0}, x^{1}\right), \dot{W}_{2}^{\frac{4}{3}, a}(\Omega)\right)_{\theta, \infty} .
$$

Since

$$
\left\{f \in W_{2}^{\frac{a}{3}, a}\left(\Omega, x^{0}, x^{1}\right)+W_{2}^{\frac{4}{3}, a}(\Omega): f\left|\partial \Omega=\frac{\partial f}{\partial x_{2}}\right| \partial \Omega=0\right\}
$$

is a complemented subspace of $W_{2}^{\frac{8}{3}, a}\left(\Omega, x^{0}, x^{1}\right)+W_{2}^{\frac{4}{3}, a}(\Omega)$ with the same projection operator, we may use [26: Theorem $1.17 .1 / 1]$ and have

$$
\begin{aligned}
& \left(\dot{W}_{2}^{\frac{8}{3}, a}\left(\Omega, x^{0}, x^{1}\right), \dot{\circ}_{2}^{\frac{4}{3}, a}(\Omega)\right)_{\theta, \infty} \\
& \quad=\left\{f \in\left(W_{2}^{\frac{8}{3}, a}\left(\Omega, x^{0}, x^{1}\right), W_{2}^{\frac{4}{3}, a}(\Omega)\right)_{\theta, \infty}: f\left|\partial \Omega=\frac{\partial f}{\partial x_{2}}\right| \partial \Omega=0\right\} .
\end{aligned}
$$


On the other hand, using (3.4), by [2: Theorem 4.2/a] we have

$$
\left(W_{2}^{\frac{8}{3}, a}(\Omega), W_{2}^{\frac{4}{3}, a}(\Omega)\right)_{\theta, \infty}=B_{2 \infty}^{\frac{a}{3}-\frac{2-a}{2}, a}(\Omega)
$$

where $\theta$ is again the number defined in (5.13). It follows from (5.15) - (5.17) and elementary properties of real interpolation that

$$
\mathcal{A}_{r}^{-1} \operatorname{maps} B_{2 \infty}^{-\frac{2-d}{2}, a}(\Omega) \text { bounded into } \stackrel{B}{B}_{2 \infty}^{\frac{8}{3}-\frac{2-d}{2}, a}(\Omega)
$$

and this completes the proof of the boundedness from the third line of (5.9).

Step 4: Let $f \in \dot{W}_{2}^{(1,2)}(\Omega)$ be an eigenfunction of $T$. Then it follows from (5.9) that $f$ belongs also to

$$
\dot{B}_{2 \infty}^{\frac{8}{3}-\frac{2-d}{2}, a}(\Omega)
$$

and so it is an eigenfunction of the operator $T$ restricted to this space. Obviously, the converse is also true. Hence the root systems considered in $\mathscr{W}_{2}^{(1,2)}(\Omega)$ and in $\stackrel{\circ}{B}_{2 \infty}^{\frac{8}{3}-\frac{2-d}{2}, a}(\Omega)$ coincide. Then the eigenvalues of $T$ considered in these spaces also coincide, inclusively their multiplicities. Using the multiplicity property (5.4) for entropy numbers and using (5.9) there exists a constant $c>0$ such that, for all $k \in \mathbb{N}$,

$$
e_{k}\left(T: \stackrel{\circ}{B}_{2 \infty}^{\frac{8}{3}-\frac{2-d}{2}, a}(\Omega) \rightarrow \stackrel{\circ}{B}_{2 \infty}^{\frac{8}{3}-\frac{2-d}{2}, a}(\Omega)\right) \leq c e_{k}\left(\operatorname{tr}_{\Gamma}:{\stackrel{\circ}{B_{2 \infty}}}_{\frac{8}{3}-\frac{2-d}{2}, a}(\Omega) \rightarrow L_{2}(\Gamma)\right)
$$

Inserting in Theorem $5.1 a=\left(\frac{4}{3}, \frac{2}{3}\right), p_{1}=p_{2}=2, q=\infty$ and $s+\frac{2-d}{2}=\frac{8}{3}-\frac{2-d}{2}$ we have from (5.18)

$$
e_{k}\left(T: \stackrel{\circ}{B}_{2 \infty}^{\frac{8}{3}-\frac{2-d}{2}, a}(\Omega) \rightarrow \dot{B}_{2 \infty}^{\frac{8}{3}-\frac{2-d}{2}, a}(\Omega)\right) \leq c k^{-\frac{1}{d}\left(d+\frac{2}{3}\right)}
$$

Estimate (4.7) is now a simple consequence of (5.19) using Carl's inequality (5.6).

5.3 Proof of estimate (4.8). Step 1: Atomic decompositions in anisotropic Besov spaces. Let $a=\left(a_{1}, a_{2}\right)$ be a given two-dimensional anisotropy and let $\mathbb{Z}^{2}$ be the lattice of all points in $\mathbb{R}^{2}$ with integer-valued components. If $\nu \in \mathbb{N}_{0}$ and $m=\left(m_{1}, m_{2}\right) \in \mathbb{Z}^{2}$, we denote $Q_{\nu m}^{a}$ the rectangle in $\mathbb{R}^{2}$ centred at $2^{-\nu a} m=\left(2^{-\nu a_{1}} m_{1}, 2^{-\nu a_{2}} m_{2}\right)$ which has sides parallel to the axes and side lengths respectively $2^{-\nu a_{1}}$ and $2^{-\nu a_{2}}$. Remark that $Q_{0 m}^{a}$ is a square with side length 1 . If $Q_{\nu m}^{a}$ is such a rectangle in $\mathbb{R}^{2}$ and $c>0$, then $c Q_{\nu m}^{a}$ is the rectangle in $\mathbb{R}^{2}$ concentric with $Q_{\nu m}^{a}$ and with side lengths respectively $c 2^{-\nu a_{1}}$ and $c 2^{-\nu a_{2}}$.

If $\beta=\left(\beta_{1}, \beta_{2}\right) \in \mathbb{N}_{0}^{2}$, the derivatives $D^{\beta}$ have the usual meaning, and if $x=$ $\left(x_{1}, x_{2}\right) \in \mathbb{R}^{2}$, then $x^{\beta}=x_{1}^{\beta_{1}} x_{2}^{\beta_{2}}$. The scalar product between the anisotropy $a=\left(a_{1}, a_{2}\right)$ and $\beta$ is $a \beta=a_{1} \beta_{1}+a_{2} \beta_{2}$. 
Definition 5.2. Let $s>0,1 \leq p \leq \infty, K \in \mathbb{R}$ and $c>1$. A function $\rho: \mathbb{R}^{2} \rightarrow \mathbb{C}$ for which there exist all derivatives $D^{\beta} \rho$ if $a \beta \leq K$ (continuous if $K \leq 0$ ) is called an anisotropic $(s, p)_{K}$-atom or simply $(s, p)$-atom, if

$$
\begin{array}{ll}
\operatorname{supp} \rho \subset c Q_{\nu m}^{a} \quad \text { for some } & \nu \in \mathbb{N} \text { and } m \in \mathbb{Z}^{2} \\
\left|D^{\beta} \rho(x)\right| \leq 2^{-\nu\left(s-\frac{2}{p}\right)} 2^{\nu a \beta} & \text { if } a \beta \leq K .
\end{array}
$$

If conditions (5.20) and (5.21) are satisfied for $\nu=0$, then $\rho: \mathbb{R}^{2} \rightarrow \mathbb{C}$ is called an anisotropic $1_{K}$-atom.

If the atom $\rho$ is located at $Q_{\nu m}^{a}$ (that means $\operatorname{supp} \rho_{\nu m}^{a} \subset c Q_{\nu m}^{a}$ with $\nu \in \mathbb{N}_{0}, m \in \mathbb{Z}^{2}$ and $c>1$ ), then we will write it $\rho_{\nu m}^{a}$.

We give some technical explanations. The value of the number $c>1$ in $(5.20)$ is unimportant. It simply makes clear that at the level $\nu$ some controlled overlapping of the supports of $\rho_{\nu m}^{a}$ must be allowed. If $K \leq 0$, then $(5.21)$ is $|\rho(x)| \leq 2^{-\nu\left(s-\frac{2}{p}\right)}$. The reason for the normalising factor in (5.21) is that there exists a constant $c>0$ such that for all these atoms we have $\left\|\rho \mid B_{p q}^{(s, a)}\left(\mathbb{R}^{2}\right)\right\| \leq c$. Hence, as in the isotropic case, atoms are normalised building blocks. This construction generalizes isotropic atoms as they are in the works of M. Frazier and B. Jawerth (see $[15,16])$.

If $0<p, q \leq \infty$, then $b_{p q}$ is the collection of all sequences $\lambda=\left\{\lambda_{\nu m} \in \mathbb{C}: \nu \in\right.$ $\mathbb{N}_{0}$ and $\left.m \in \mathbb{Z}^{2}\right\}$ such that

$$
\left\|\lambda \mid b_{p q}\right\|=\left(\sum_{\nu=0}^{\infty}\left(\sum_{m \in \mathbf{Z}^{2}}\left|\lambda_{\nu m}\right|^{p}\right)^{\frac{q}{p}}\right)^{\frac{1}{q}}
$$

(with the usual modification if $p=\infty$ and/or $q=\infty$ ) is finite. Clearly $b_{p q}$ is a quasiBanach space.

Theorem 5.3. Let $a=\left(a_{1}, a_{2}\right)$ a given anisotropy with $a_{2} \leq a_{1}$. Let $s>0$, $1 \leq p \leq \infty, 0<q \leq \infty$, and let $K \in \mathbb{R}$ such that $K \geq a_{1}+s$. Then $g \in S^{\prime}\left(\mathbb{R}^{2}\right)$ belongs to $B_{p q}^{s, a}\left(\mathbb{R}^{2}\right)$ if and only if it can be represented as

$$
g=\sum_{\nu=0}^{\infty} \sum_{m \in \mathbf{Z}^{2}} \lambda_{\nu m} \rho_{\nu m}^{a} \quad \text { (convergence being in } S^{\prime}\left(\mathbb{R}^{2}\right) \text { ) }
$$

where $\rho_{\nu m}^{a}$ are anisotropic $1_{K}$-atoms $(\nu=0)$ or anisotropic $(s, p)_{K}$-atoms $(\nu \in \mathbb{N})$ and $\lambda \in b_{p q}$ where $\lambda=\left\{\lambda_{\nu m}: \nu \in \mathbb{N}_{0}\right.$ and $\left.m \in \mathbb{Z}^{2}\right\}$. Furthermore, inf $\left\|\lambda \mid b_{p q}\right\|$ where the infimum is taken over all admissible representations (5.22) is an equivalent quasi-norm in $B_{p q}^{s, a}\left(\mathbb{R}^{2}\right)$.

This is a weak version of [12: Theorem 3.3] which will be needed below. We will refer to the above theorem as to the atomic decomposition theorem in anisotropic function spaces since it generalises to the anisotropic case the well known results of $M$. Frazier and B. Jawerth from $[15,16]$.

Step 2: Approximation numbers and eigenvalues of operators acting in Hilbert spaces. We recall the definition of the approximation numbers. Let $B_{1}$ and $B_{2}$ be 
two quasi-Banach spaces and let $U \in L\left(B_{1}, B_{2}\right)$. Then given any $k \in \mathbb{N}$, the $k$-th approximation number $\alpha_{k}(U)$ of $U$ is defined by

$$
\alpha_{k}(U)=\inf \left\{\|U-L\|: L \in L\left(B_{1}, B_{2}\right) \text { and } \operatorname{rank} L<k\right\}
$$

where $\operatorname{rank} L$ is the dimension of the range of $L$. Usually the approximation numbers are denoted $a_{k}(U)$. The above notation is used only to avoid any possible confusion between these numbers and the anisotropy $a=\left(a_{1}, a_{2}\right)$. We do not want to discuss here properties of approximation numbers, this is done in [8: Lemma 1.3.1/2 and Remark 1.3.1/6] and [7: Section II.2.3]. We only want to mention that approximation numbers have important connections with eigenvalues, the picture being clearest in a Hilbert space setting.

Theorem 5.4. Let $H$ be a Hilbert space and let $U \in L(H)$ be a compact, non. negative and self-adjoint operator. Then the approximation numbers $\alpha_{k}(U)$ of $U$ coincide with its eigenvalues (ordered as in (5.5)).

A proof can be found in [7: Section II.5.10] (see also [8: page 21]).

Step 3: If one applies Theorem 5.4, then estimate (4.8) is a simple consequence of the next proposition.

Proposition 5.5. Let $\Omega$ and $d$ as above, let $\Gamma$ be proper and let $T$ be the compact, non-negative, self-adjoint operator in $\mathrm{W}_{2}^{(1,2)}(\Omega)$ defined in (4.4). There exists a constant $c>0$ such that the approximation numbers $\alpha_{k}(\sqrt{T})$ of $\sqrt{T}=T^{\frac{1}{2}}$ can be estimated by

$$
\alpha_{k}(\sqrt{T}) \geq c k^{-\frac{1}{d}\left(\frac{d}{2}+\frac{1}{3}\right)} \quad(k \in \mathbb{N}) .
$$

Step 4: Proof of Proposition 5.5. Let $x^{j, l}$ be the centre of the rectangle $R_{j l}$ of side lengths $r_{1}^{j, l}$ and $r_{2}^{j, l}$, and let $N_{j} \sim 2^{j d}$ having the same meaning as in Theorem 2.1. Since $\Gamma \subset \Omega$, it is clear that there exists a $j_{0} \in \mathbb{N}_{0}$ such that for any $j \geq j_{0}$ the rectangles $R_{j l}\left(l=1, \ldots, N_{j}\right)$ are contained in $\Omega$. Let $\varphi$ a non-negative $C^{\infty}$ function on $\mathbb{R}^{2}$ with support in $\left\{x \in \mathbb{R}^{2}:\left|x_{1}\right|<1\right.$ and $\left.\left|x_{2}\right|<1\right\}$. We may assume $|\varphi(x)| \geq \delta>0$ if $\left|x_{1}\right| \leq \kappa$ and $\left|x_{2}\right| \leq \kappa$ where $0<\kappa<1$ is the number from Definition 2.3 of a proper set. If

$$
\varphi_{j l}(x)=\varphi\left(\frac{2\left(x_{1}-x_{1}^{j, l}\right)}{r_{1}^{j, l}}, \frac{2\left(x_{2}-x_{2}^{j, l}\right)}{r_{2}^{j, l}}\right)
$$

then $\operatorname{supp} \varphi_{j l} \subset R_{j l}$. Furthermore, there exist two constants $c_{1}, c_{2}>0$ such that

$$
c_{1} 2^{-j \frac{d}{2}}\left(\sum_{l=1}^{N_{j}}\left|c_{j l}\right|^{2}\right)^{\frac{1}{2}} \leq\left\|\sum_{l=1}^{N_{j}} c_{j l} \varphi_{j l} \mid L_{2}(\Gamma)\right\| \leq c_{2} 2^{-j \frac{d}{2}}\left(\sum_{l=1}^{N_{j}}\left|c_{j l}\right|^{2}\right)^{\frac{1}{2}}
$$

for any complex numbers $c_{j l}$ and for any $j \geq j_{0}$ and $l=1, \ldots, N_{j}$. Indeed, using (2.7) 
we have

$$
\begin{aligned}
\left\|\sum_{l=1}^{N_{j}} c_{j l} \varphi_{j l} \mid L_{2}(\Gamma)\right\|^{2} & =\sum_{l=1}^{N_{j}} \int_{\Gamma \cap R_{j l}}\left|c_{j l}\right|^{2}\left|\varphi_{j l}(\gamma)\right|^{2} d \mu(\gamma) \\
& \geq \sum_{l=1}^{N_{j}}\left|c_{j l}\right|^{2} \delta^{2} \mu\left(\Gamma \cap \kappa R_{j l}\right) \\
& \geq c 2^{-j d} \sum_{l=1}^{N_{j}}\left|c_{j l}\right|^{2}
\end{aligned}
$$

and this is the first inequality in (5.24). The second part of (5.24) is a simple consequence of (2.5).

Remark now that if $j \geq j_{0}$ is fixed and $l \in\left\{1, \ldots, N_{j}\right\}$, then $2^{-j \frac{1}{3}} \varphi_{j l}$ is an anisotropic $\left(\frac{4}{3}, 2\right)$ atom in $\stackrel{\circ}{W}_{2}^{(1,2)}(\Omega)$. Hence, using (4.3) and the atomic decomposition theorem in anisotropic Besov spaces with $s=\frac{4}{3}$ and $p=q=2$ we have

$$
\left\|g_{j} \mid \dot{W}_{2}^{(1,2)}(\Omega)\right\| \leq c 2^{j \frac{1}{3}}\left(\sum_{l=1}^{N_{j}}\left|c_{j l}\right|^{2}\right)^{\frac{1}{2}}
$$

for any function $g_{j}$ of the type

$$
g_{j}=\sum_{l=1}^{N_{j}} c_{j l} \varphi_{j l}=2^{j \frac{1}{3}} \sum_{l=1}^{N_{j}} c_{j l}\left(2^{-j \frac{1}{3}} \varphi_{j l}\right)
$$

the constant $c>0$ in (5.25) being independent of $j, l$ and of the complex numbers $c_{j l}$. By (5.2), (5.24) and (5.25) we find a constant $c_{0}$ such that

$$
\left\|\sqrt{T} g_{j}\left|\stackrel{\circ}{W}_{2}^{(1,2)}(\Omega)\left\|\sim 2^{-j \frac{d}{2}}\left(\sum_{l=1}^{N_{j}}\left|c_{j l}\right|^{2}\right)^{\frac{1}{2}} \geq c_{0} 2^{-j\left(\frac{d}{2}+\frac{1}{3}\right)}\right\| g_{j}\right| \dot{W}_{2}^{(1,2)}(\Omega)\right\| .
$$

There exists an operator $L=L\left(N_{j}\right)$ in $\stackrel{\circ}{W}_{2}^{(1,2)}(\Omega)$ with rank $L<N_{j}$ such that

$$
\alpha_{N_{j}}(\sqrt{T}) \geq\|\sqrt{T}-L\|-\frac{1}{2} c_{0} 2^{-j\left(\frac{d}{2}+\frac{1}{3}\right)}
$$

where $c_{0}$ is the number from the last line in (5.27). We may assume that the dimension of the span of the admitted functions $g_{j}$ in (5.26) is larger than $N_{j} \sim 2^{j d}$. Then we find a function $g_{j}$ of type $(5.26)$ in $\dot{W}_{2}^{(1,2)}(\Omega)$ such that $\left\|g_{j} \mid \dot{W}_{2}^{(1,2)}(\Omega)\right\|=1$ and $L g_{j}=0$. It follows

$$
\alpha_{N_{j}}(\sqrt{T}) \geq\left\|\sqrt{T} g_{j}-L g_{j} \mid \dot{W}_{2}^{(1,2)}(\Omega)\right\|-\frac{1}{2} c_{0} 2^{-j\left(\frac{d}{2}+\frac{1}{3}\right)} \geq c_{0} 2^{-j\left(\frac{d}{2}+\frac{1}{3}\right)}
$$

where $c_{0}>0$ is independent of $j$. Using elementary properties of approximation numbers it is easy to see that (5.28) implies (5.23) and this completes the proof of the theorem 
A final remark. Probably the most important result stated in Theorem 4.2 is estimate (4.7). We want to point out that in the proof of (4.7) the key role is played by the mapping property in the third line of (5.9), more precisely by the fact that for sufficiently large $r$ the operator $\mathcal{A}_{r}^{-1}$ maps the anisotropic Besov space $B_{2 \infty}^{-\frac{2-d}{2}, a}(\Omega)$ bounded into

$$
\stackrel{\circ}{B}_{2 \infty}^{\frac{8}{3}-\frac{2-d}{2}, a}(\Omega)=\left\{f \in B_{2 \infty}^{\frac{8}{3}-\frac{2-d}{2}, a}(\Omega): f\left|\partial \Omega=\frac{\partial f}{\partial x_{2}}\right| \partial \Omega=0\right\} .
$$

This was obtained as a consequence of the results from [28] using some interpolation results from $[2,3]$.

We think this remark paves the way to further investigations. One can try to replace the model operator $\mathcal{A}_{r}$ by a more general (semi-elliptic) operator, the space $\dot{W}_{2}^{(1,2)}(\Omega)$ by another anisotropic function space but the considered anisotropic fractal should be chosen having the same anisotropy as the differential operator. Some steps in this direction were done in [13] were the surrounding domain of the fractal was the whole $\mathbb{R}^{2}$.

\section{References}

[1] Bernardi, M. P. and C. Bondioli: On some dimension problems for self-affine fractals. $Z$. Anal. Anw. (to appear).

[2] Besov, O. V.: Interpolation of spaces of differentiable functions on a domain (in Russian). Trudy Mat. Inst. Steklov 214 (1996), $59-82$.

[3] Besov, O. V.: Interpolation and embeddings of generalized function spaces $B_{p q}^{s}, F_{p q}^{s}$ on a domain (in Russian). Trudy Mat. Inst. Steklov 219 (1997), 80 - 102.

[4] Besov, O. V., Il'in, V. P. and S. M. Nikol'skij: Integral Representations of Functions and Embedding Theorems (in Russian). Moscow: Nauka 1975.

[5] Bricchi, M.: On the measure of Hutchinson for regular anisotropic fractals. Personal communication, Pavia 1998.

[6] Carl, B.: Entropy numbers, s- numbers and eigenvalue problems. J. Funct. Anal. 41 (1981), 290 - 306.

[7] Edmunds, D. E. and W. D. Evans: Spectral Theory and Differential Operators. Oxford: Univ. Press 1987.

[8] Edmunds, D. E. and H. Triebel: Function Spaces, Entropy Numbers, Differential Operators. Cambridge: Univ. Press 1996.

[9] Edmunds, D. E. and H. Triebel: Spectral theory for isotropic fractal drums. C. R. Acad. Sci. Paris 326 (1998), $1269-1274$.

[10] Falconer, K. J.: The Geometry of Fractal Sets. Cambridge: Univ. Press 1985.

[11] Falconer, K. J.: Fractal Geometry. Chichester: Wiley 1990.

[12] Farkas, W.: Atomic and subatomic decompositions in anisotropic function spaces. Math. Nachr. (to appear).

[13] Farkas, W.: Eigenvalue distribution of some fractal semi-elliptic differential operators. Preprint. Jena: Univ. 1998 (submitted). 
[14] Farkas, W. and H. Triebel: The distribution of eigenfrequencies of anisotropic fractal drums. J. London Math. Soc. (to appear).

[15] Frazier, M. and B. Jawerth: Decomposition of Besov spaces. Indiana Univ. Math. J. 34 (1985), 777 - 799.

[16] Frazier, M. and B. Jawerth: $A$ discrete transform and decomposition of distribution spaces. J. Funct. Anal. 93 (1990), $34-170$.

[17] Hutchinson, J. E.: Fractals and self similarity. Indiana Univ. Math. J. 30 (1981), 713 747.

[18] Jonsson, A. and H. Wallin: Function spaces on subsets of $\mathbb{R}^{n}$, Math. Reports Vol. 2/Part 1. London: Harwood Acad. Publ. 1984.

[19] Mandelbrot, B.: Self-affine fractal sets. In: Fractals in Physics (eds: L. Pietronero and E. Tosatti). Amsterdam: Elsevier Sci. Publ. 1986, pp. 3 - 28.

[20] McMullen, C.: The Hausdorff dimension of general Sierpinski carpets. Nagoya Math. J. 96 (1984), 1 - 9.

[21] Naimark, K. and M. Solomyak: On the eigenvalue behaviour for a class of operators related to self-similar measures on $\mathbb{R}^{d}$. C. R. Acad. Sci. Paris 319 (1994), $837-842$.

[22] Naimark, K. and M. Solomyak: The eigenvalue behaviour for the boundary value problems related to self-similar measures on $\mathbb{R}^{d}$. Math. Res. Letters 2 (1995), $279-298$.

[23] Nikol'skij, S. M.: Approximation of Functions of Several Variables and Embedding Theorems (in Russian). Moscow: Nauka 1977.

[24] Schmeißer, H.-J. and H. Triebel: Topics in Fourier Analysis and Function Spaces. Leipzig: Geest \& Portig 1987.

[25] Shevchik, V.: Spectral properties of some semi-elliptic operators in $L_{p}$-spaces. Math. Nachr. (to appear).

[26] Triebel, H.: Interpolation Theory, Function Spaces, Differential Operators. Amsterdam: North Holland 1978.

[27] Triebel, H.: Theory of Function Spaces. Leipzig: Geest \& Portig 1983.

[28] Triebel, H.: A priori estimates and boundary value problems for semi-elliptic differential equations: a model case. Comm. Part. Diff. Equ. 8 (1983), 1621-1644.

[29] Triebel, H.: Anisotropic function spaces. Part I: Hardy's inequality, decompositions. Analysis Mathematica 10 (1984), 53 - 77.

[30] Triebel, H.: Theory of Function Spaces II. Basel: Birkhäuser 1992.

[31] Triebel, H.: Higher Analysis. Leipzig: Barth Verlag 1992.

[32] Triebel, H.: Fractals and Spectra. Basel: Birkhäuser 1997.

[33] Triebel, H. and H. WinkelvoB: A Fourier analytical characterization of the Hausdorff dimension of a closed set and of related Lebesgue spaces. Studia Math. 121 (1996), 149 166.

[34] Yamazaki, M.: A quasi-homogeneous version of paradifferential operators. Part I: Boundedness on spaces of Besov type. J. Fac. Sci. Univ. Tokyo, Sect. I. A: Math. 33 (1986), $131-174$. 